\title{
Impact of tetrabutylammonium, iodide and triiodide ions conductivity in polyacrylonitrile based electrolyte on DSSC performance
}

\begin{abstract}
Gel polymer electrolytes (GPEs) with polyacrylonitrile (PAN)-based polymer, ethylene carbonate (EC) and propylene carbonate (PC) plasticizers, and different amounts of tetrabutylammonium iodide (TBAI) salt and iodine (I2) have been prepared and used in dyesensitized solar cells (DSSCs). The maximum room temperature conductivity of $5.14 \mathrm{mS} \mathrm{cm}-1$ is obtained for electrolyte with a composition of $8 \mathrm{wt} \%$ PAN-30 wt\% EC-30 wt\% PC-30 wt\% TBAI-2 wt $\%$ I2 (S3 electrolyte) which influenced by the highest charge carrier density of 7.93 $\times 1020 \mathrm{~cm}-3$ estimated from fitting the impedance Nyquist plot. The DSSC fabricated with S3 electrolyte revealed the highest power conversion efficiency of 3.45\% with open-circuit voltage (Voc) of $582 \mathrm{mV}$ and short-circuit current density (Jsc) of $12.9 \mathrm{~mA} \mathrm{~cm}-2$. The incident photon-to-current conversion efficiency of the DSSC with highest efficiency is $54.01 \%$. The electrical impedance spectroscopy of the same cell shows the lowest series resistance indicating the superiority of electrolyte charge transport characteristics in DSSC. In addition, electron transfer time constant and electron recombination time, charge collection efficiency, electron diffusion coefficient and diffusion length of DSSC fabricated with GPEs prepared have been estimated by intensity-modulated photocurrent spectroscopy and intensity-modulated photovoltage spectroscopy techniques. The DSSC with highest efficiency shows lowest of $34.46 \mathrm{~ms}$ and highest of $90.41 \mathrm{~ms}$ due to the huge amount of TBA+ ions that covered the surface area of mesoporous TiO2. The of 0.62 , D of $4.00 \times 10-5 \mathrm{~cm} 2 \mathrm{~s}-1$ and of $19.02 \mu \mathrm{m}$ further support the photovoltaic efficiency of DSSC.
\end{abstract}

Keyword: Dye-sensitized solar cells; Electrical transport properties; Electron recombination time; Electron transfer time constant; Diffusion length 